\title{
Lattice Microarchitecture for Bone Tissue Engineering from Calcium Phosphate Compared to Titanium
}

\author{
Tse-Hsiang Chen, MS, Chafik Ghayor, PhD, Barbara Siegenthaler, PhD, Felix Schuler, MS, \\ Jasmine Rüegg, MS, Michael De Wild, $\mathrm{PhD}^{2}$, and Franz E. Weber, $\mathrm{PhD}^{1,3,4}$
}

Additive manufacturing of bone tissue engineering scaffolds will become a key element for personalized bone tissue engineering in the near future. Several additive manufacturing processes are based on extrusion where the deposition of the filament will result in a three-dimensional lattice structure. Recently, we studied diverse lattice structures for bone tissue engineering realized by laser sintering of titanium. In this work, we used lithographybased ceramic manufacturing of lattice structures to produce scaffolds from tricalcium phosphates (TCP) and compared them in vivo to congruent titanium scaffolds manufactured with the identical computer-aided design data to look for material-based differences in bony healing. The results show that, during a 4-week period in a noncritical-size defect in a rabbit calvarium, both scaffolds with the identical microarchitecture performed equally well in terms of bony regeneration and bony bridging of the defect. A significant increase in both parameters could only be achieved when the TCP-based scaffolds were doped with bone morphogenetic protein-2. In a critical-size defect in the calvarial bone of rabbits, however, the titanium scaffold performed significantly better than the TCP-based scaffold, most likely due to its higher mechanical stability. We conclude that titanium and TCP-based scaffolds of the same microarchitecture perform equally well in terms of bone regeneration, provided the microarchitecture meets the mechanical demand at the site of implantation.

Keywords: lithography, calcium phosphate, titanium, bone regeneration, bone repair, lattice architecture, additive manufacturing, osteoconduction

\section{Impact Statement}

The microarchitecture of bone substitutes is an important factor for successful bone regeneration via osteoconduction; as is the choice of the material. To dissect osteoconduction determined by bony bridging and bony regeneration of a defect in material and microarchitectural elements, we produced scaffolds with congruent microarchitecture from titanium and TCP by additive manufacturing. For the first time we could show that for open porous lattice microarchitectures, titanium and TCP based scaffolds are equally osteoconductive, if they meet the mechanical demands of the defect.

\section{Introduction}

A UTOLOGOUS BONE IS still the gold standard bone substitute and after blood the most frequent transplanted material in clinics worldwide. ${ }^{1}$ Since many years, bone tissue engineering is focused on substitution of autografts. However, due to its limited supply, additional pain at the second operating site, and donor site morbidity, smarter grafting methods are needed that would overcome these issues. ${ }^{2}$ In recent years, special emphasis has been given to the use of mesenchymal stem cells $^{3-6}$ and bone morphogenetic proteins $\left(\mathrm{BMPs}^{7-9}\right)$ to further improve the performance of synthetic bone substitute materials. The first one, however, is costly and time-consuming and the second one has caused well-documented side effects reported over the last decade. ${ }^{10}$ Therefore, the search for a bone substitute, which can compete with autologous bone, is still ongoing.

\footnotetext{
${ }^{1}$ Oral Biotechnology and Bioengineering, Center of Dental Medicine, University of Zurich, Zurich, Switzerland.

${ }^{2}$ School of Life Sciences, Institute for Medical and Analytical Technologies, University of Applied Sciences Northwestern Switzerland, Muttenz, Switzerland.

${ }_{4}^{3} \mathrm{CABMM}$, Center for Applied Biotechnology and Molecular Medicine, University of Zurich, Zurich, Switzerland.

${ }^{4}$ Zurich Center for Integrative Human Physiology (ZIHP), University of Zurich, Zurich, Switzerland.

(C) Tse-Hsiang Chen et al. 2018; Published by Mary Ann Liebert, Inc. This Open Access article is distributed under the terms of the Creative Commons Attribution Noncommercial License (http://creativecommons.org/licenses/by-nc/4.0/) which permits any noncommercial use, distribution, and reproduction in any medium, provided the original author(s) and the source are cited.
} 
From the material point of view, bone is a composite from collagen and calcium phosphate $(\mathrm{CaP})$ deposited as hydroxyapatite. Therefore, CaP-based ceramics are the most widely applied synthetic biomaterials for repair and regeneration of damaged and diseased bone. ${ }^{11} \mathrm{CaP}$ not only resembles natural bone mineral but is also bioactive in terms of biocompatibility, osteoconductivity, and osteoinductivity. ${ }^{12}$ Its biocompatibility is evident by the direct bone bonding capacity, its osteoconduction by the advancement of bone deposition on the surface, and its osteoinduction by a limited capacity to induce de novo bone formation close to $\mathrm{CaP}$ implants. All these bioactivities of $\mathrm{CaP}$ are attributed to the chemical composition, surface topography, macroporosity/microporosity, and the dissolution kinetics of the calcium $\left(\mathrm{Ca}^{2+}\right)$ and phosphate $\left(\mathrm{PO}_{4}{ }^{3-}\right)$ ions, as reviewed in Ref. ${ }^{12}$

To mimic cancellous autologous bone and allow bone ingrowth, CaP-based scaffolds are produced with a certain porosity as reviewed in Ref. ${ }^{13}$ The ideal pore size was found to be between 0.3 and $0.5 \mathrm{~mm}$. A more recent article reported that no difference in bone regeneration was seen up to a poresize of $1.2 \mathrm{~mm} .{ }^{14}$ In all these porous CaP-based scaffolds used for the identification of the ideal pore dimension, the pores were introduced by porogens causing a random distribution of the pores and no direct control over the size of the interconnections between the pores. To overcome these limitations of all those studies, the application of additive manufacturing produces scaffolds with defined pore sizes, pore locations, microarchitecture, and defined connections between pores. The process of additive manufacturing creates the scaffold layer upon layer for example by stereolithography or selective laser sintering. ${ }^{15}$ In three-dimensional printing, another methodology in additive manufacturing, the object is formed in a powder bed by the deposition of a liquid binder through inkjet heads ${ }^{16}$ This methodology has already been applied for ceramics, ${ }^{17}$ but certain limitations of design freedom exist to allow the release of powder entrapped inside the scaffold during the production in the powder bed. ${ }^{18}$ In recent years, we aimed to study the ideal microarchitecture of bone substitutes by selective laser sintering where a high-intensity laser beam was used to build scaffolds in a titanium powder bed. ${ }^{19,20} \mathrm{We}$ found open titanium lattice structures to allow a fast defect bridging, which could not be accelerated by the double delivery of BMPs and their enhancer. ${ }^{21}$ The disadvantage of using a titanium-based bone substitute is its nondegradability. Therefore, we looked for an additive manufacturing system to produce filigree structures from ceramics.

In this study, we applied the CeraFab 7500 (Lithoz, Vienna, Austria) for the production of lattice structures from tricalcium phosphates (TCP), where fine structures are produced from a TCP slurry by a lithography-based printing process in an upside-down process. In this study, we report on the accuracy of the system and the morphology of the sintered material. Moreover, we studied its performance in vivo in comparison to titanium scaffolds produced by selective laser melting out of the same digital information file data (STL: standard triangulation language) in a noncritical calvarial defect and a critical calvarial defect model in rabbits. By doing so, we were able to compare both widely accepted materials in bone tissue engineering: titanium and TCP, for osteoconduction quantified by bony bridging and bony regeneration of the defects with scaffolds of identical microarchitecture.
The aims of this study were to characterize a lithographybased additively manufactured TCP scaffold and compare it to a titanium-based scaffold with identical microarchitecture in the context of bone tissue engineering. Moreover, we wanted to compare the osteoconductivity of a lattice microarchitecture realized with two widely accepted, but very distinct materials to test for material-dependent effects on osteoconduction.

\section{Methods}

\section{Implant production}

Titanium implants were produced as previously reported..$^{19,20}$ For critical-size defects, lattice implants with $\emptyset 15 \mathrm{~mm}$ outer diameter were produced; for noncritical-size defects, $\varnothing 6 \mathrm{~mm}$ scaffolds were produced. The microarchitecture of all implants consists of orthogonal struts of ca. $300 \mu \mathrm{m}$ diameter separated by ca. $500 \mu \mathrm{m}$ wide interconnected channels. The TCP scaffolds were produced with a TCP slurry LithaBone ${ }^{\mathrm{TM}}$ TCP $200\left(\mathrm{Li}^{-}\right.$ thoz, Vienna, Austria), solidified in the CeraFab 7500 system (Lithoz) by the exposure of the photoactive polymer from the slurry to a blue LED light in a layer-by-layer manner. ${ }^{22}$ The so-formed TCP green part was composed of layers of $25 \mu \mathrm{m}$ thickness and a resolution of $50 \mu \mathrm{m}$ in the $x / y$-plane. Upon cleaning of the green part with LithaSol $20^{\mathrm{TM}}$ (Lithoz) and pressurized air, they underwent a thermal treatment to decompose the polymeric binder and densify them by sintering. The final stage of the sintering procedure covered a dwelling time of $3 \mathrm{~h}$ at $1100^{\circ} \mathrm{C}$, as recommended by the manufacturer of the slurry. The sintered scaffolds were transferred into a sterile bench, packed for transportation into the operation theater, and used as bone substitute implant without further sterilization.

\section{Scanning electron microscopy}

The scaffolds were examined using a Zeiss Supra V50 scanning electron microscope (SEM) (Carl Zeiss, Oberkochen, Germany). Scanning was performed under acceleration voltage of $12 \mathrm{kV}$ with a distance of the sample to the detector of $9.5 \mathrm{~cm}$.

\section{Surgical procedure}

Twenty-four adult (12 months old) New Zealand White rabbits were used in this study. The animals' weights were between 3.5 and $4.0 \mathrm{~kg}$ and they were fed a standard laboratory diet. The procedures was evaluated and accepted by the local authorities (108/2012 and 114/2015). To initiate the operation, the animals were anesthetized by an injection of $65 \mathrm{mg} / \mathrm{kg}$ ketamine and $4 \mathrm{mg} / \mathrm{kg}$ xylazine and maintained under anesthesia with isoflurane/ $\mathrm{O}_{2}$. After disinfection, an incision from the nasal bone to the mid-sagittal crest was made, the soft tissue deflected, and the periosteum removed. Next, for noncritical-size defects, four evenly distributed 6-mm-diameter craniotomy defects were prepared with a trephine bur under copious irrigation with sterile saline in the operation field. Then, all defects were completed with a rose burr $(1 \mathrm{~mm})$ to preserve the dura. Next, all the defects were flushed with saline solution to remove remaining debris and the implants applied by gentle press fitting. Each of the animals received four different treatment modalities. The treatment modalities were assigned at random for the 
first animal, and thereafter, cyclic permuted clockwise. The treatments were grouped for titanium, TCP, and TCP/BMP. For the critical-size defects, a central 15-mm-diameter defect was generated as reported recently. ${ }^{21}$ After the completion of implant placement, the soft tissues were closed with interrupted sutures. Four weeks after operation for the $6 \mathrm{~mm}$ defects and 16 weeks for the critical-size defects, the rabbits were placed under general anesthesia and sacrificed by an overdose of pentobarbital. The cranium containing all four craniotomy sites was removed and placed in $40 \%$ ethanol. Embedding was performed as previously reported. ${ }^{20}$

\section{Histomorphometry}

The evaluation of all implants was performed from the middle section using image analysis software (Image-Pro Plus ${ }^{\circledR}$; Media Cybernetic, Silver Springs, MD). The area of interest (AOI) was defined by the $6 \mathrm{~mm}$, respectively, $15 \mathrm{~mm}$ defect dimension and the height of the implant. We determined the area of new bone in the AOI as percent of bone and bony integrated scaffold in the AOI (bony area, \%). For the empty control value, the average area occupied by all scaffolds was taken into account, since the height of the implants exceeds the thickness of the calvarial bone.

\section{Bone bridging}

The determination of bony bridging was performed as reported earlier. ${ }^{23,24}$ In brief, areas with bone tissue within the defect margin were projected onto the $x$-axis. Next, the stretches of the $x$-axis with projected bone tissue were summed up and related to the defect width of $6 \mathrm{~mm}$ (noncritical size), respectively $15 \mathrm{~mm}$ (critical size). Bone bridging is given in percentage of the defect width $(6 \mathrm{~mm}$, respectively $15 \mathrm{~mm}$ ) where bone formation had occurred.

\section{Statistics}

The primary analysis unit was the animal. For all parameters tested, the treatment modalities were compared with a Kruskal-Wallis test, followed by pairwise comparison of treatment modalities with the Mann-Whitney test for dependent data (IBM SPSS v.19). $p$-Values are displayed in the graphs and significance was set at a limit of $p<0.05$. Data from seven to nine different rabbits are presented for each group for the noncritical-size defect and six for the critical-size ones. Values are reported in the text by mean \pm standard deviation or displayed in graphs as median \pm lower/upper quartile.
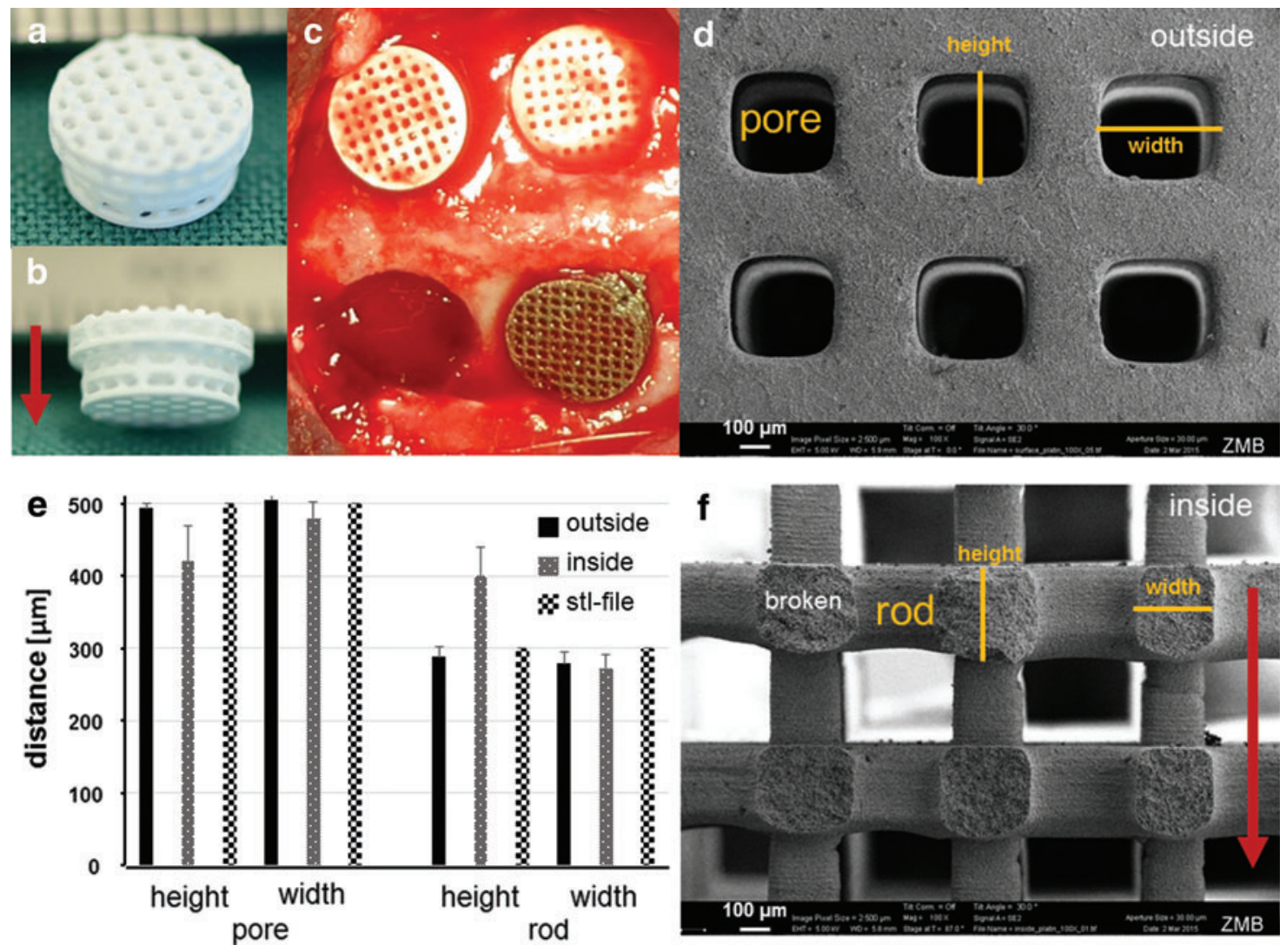

FIG. 1. Characterization of a TCP scaffold for noncritical-size defects. (a, b) Top and side view of a TCP scaffold (lower part is $6 \mathrm{~mm}$ in diameter). Building direction is indicated by the red arrow. (c) Intraoperative view of TCP and titanium scaffolds after placement into the cranial defects. The empty defect (lower left) serves as control. (d) Electron microscopical view of the outer part of the scaffold. A pore and its height and width are indicated in orange. (e) Comparison of pore and rod height and width from outside, inside, and in silico (STL file). (f) Electron microscopical top view of the inner (broken) part of the scaffold. A rod and rod height and width are indicated in orange. The red arrow illustrates the building direction. Scales are provided. TCP, tricalcium phosphates. Color images available online at www.liebertpub.com/tea 


\section{Results}

Characterization of the TCP-based scaffolds and comparison to the virtual planning

After sintering, the scaffolds appeared white, suggesting that all the binder material was removed during the sintering process, including a 3 -h sintering step at $1100^{\circ} \mathrm{C}$ (Fig. 1a, b). Next, we compared the produced scaffolds with the digital information file data put down in an STL file. In terms of precision, we evaluated the height and width of the pores and rods from the outer surface of the scaffold in top view and from the cross-section of the inner part as illustrated (Fig. 1d, f). The comparison of the aforementioned dimensions taken from the outside and the inner portion of the scaffold in comparison with the STL dimensions revealed that there are deviations in the building direction (see arrow in Fig. 1a, f), listed as pore height and rod height (Fig. 1e). These deviations arose since in these initial studies, no compensation parameters were used during the printing process; hence, a higher cure depth in $z$-direction was obtained than targeted in the CAD. SEM (Fig. 2) revealed that upon sintering, the TCP particles have partially fused and the scaffold appears to be highly microporous with pores between 2 and $4 \mu \mathrm{m}$ in diameter.

\section{In vivo evaluation and comparison of TCP to titanium}

One aim of this study was to compare the effect of the bone substitute material (TCP or titanium) on bony bridging and bony regenerated area in case the bone substitute had the identical lattice microarchitecture. In a first series of experiments, we compared bony bridging and bone formation between empty defects, titanium, TCP, and TCP scaffolds loaded with $10 \mu \mathrm{g}$ BMP-2 in noncritical-size defects. Since our model consists of four defects, the titanium implant with BMP-2 was omitted and the new TCP implant with BMP-2 was tested. The architecture of all scaffolds was identical because they were built with the same STL file.
The histologies of the middle section (Fig. 3) revealed that bone formation had occurred during the 4-week period throughout all implants, irrespective of the material.

For quantification, the extent of bony bridging and bone formation in the defect was determined (Fig. 4). In empty defects, $29.28 \% \pm 19.16 \%$ of the middle section was bridged, $71.29 \% \pm 23.95 \%$ for titanium, $89.28 \% \pm 18.45 \%$ for TCP, and $96.87 \% \pm 6.20 \%$ for TCP plus BMP-2, respectively. In the AOI, the percentage of bony regeneration in the middle section was $16.40 \% \pm 6.79 \%$ for empty defects, $39.00 \% \pm$ $9.14 \%$ for titanium, $56.94 \% \pm 19.08 \%$ for TCP, and $73.13 \% \pm$ $13.94 \%$ for TCP plus BMP-2, respectively. For both measures, all scaffolds performed better than the empty defects. No significant difference existed between titanium and TCP scaffolds. Only if doped with $10 \mu \mathrm{g}$ BMP-2, the TCP-BMP scaffold performed significantly better than the titanium scaffold, both in terms of bony bridging $(p=0.008)$ and bony regenerated area $(p=0.001)$. This result also suggests that our additively manufactured TCP scaffolds could serve as BMP-2 delivery vehicle and combine osteoconduction with osteoinduction.

\section{Critical-size defect}

To compare identical lattice-based microarchitectures from titanium and TCP in more detail we, next, moved to a criticalsize defect in the calvarial bone of rabbits. The scaffolds for this aspect were produced as mentioned before (Fig. 5a), with the diameter of the lower part of $15 \mathrm{~mm}$ adjusted to criticalsize defects ${ }^{21}$ (Fig. 5b). The samples were harvested 16 weeks after implantation. Histology revealed that the majority of the defect was bridged, although the scaffolds appeared partially disintegrated (Fig. 5c). The reason for this behavior is most likely related to the high microporosity of the TCP samples. This microporosity on the one hand enables rapid resorption of the materials, but on the other hand also increases the brittleness of the resulting scaffolds. This brittle behavior in combination with very delicate struts and with
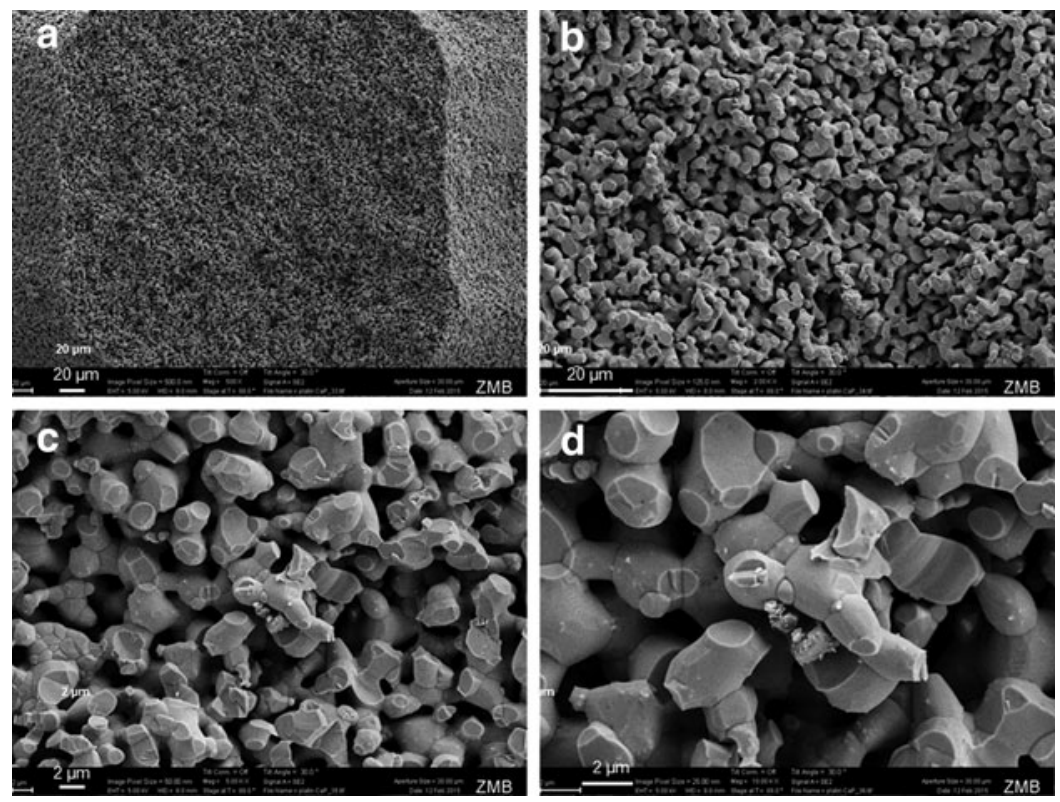

FIG. 2. SEM of a broken TCP rod. (a-d) Shows the final scaffold after sintering, Scales are provided in the panels. TCP particles and micropores appear $2-4 \mu \mathrm{m}$ in diameter. SEM, scanning electron microscopy. 
FIG. 3. Histological sections from the middle of the noncritical-size defect. (a-d) For all scaffolds and the untreated control, a histological section from 4 weeks postoperatively is shown. (a) Empty control defect; (b) titanium scaffold; (c) TCP scaffold with $10 \mu \mathrm{g}$ BMP-2; (d) TCP scaffold. Scale bars indicate $1 \mathrm{~mm}$. Original magnifications were 100 fold. Bone appears as grayish purple to purple, titanium as dark black, and TCP as grayish black. BMP, bone morphogenetic protein. Color images available online at www.liebertpub.com/tea

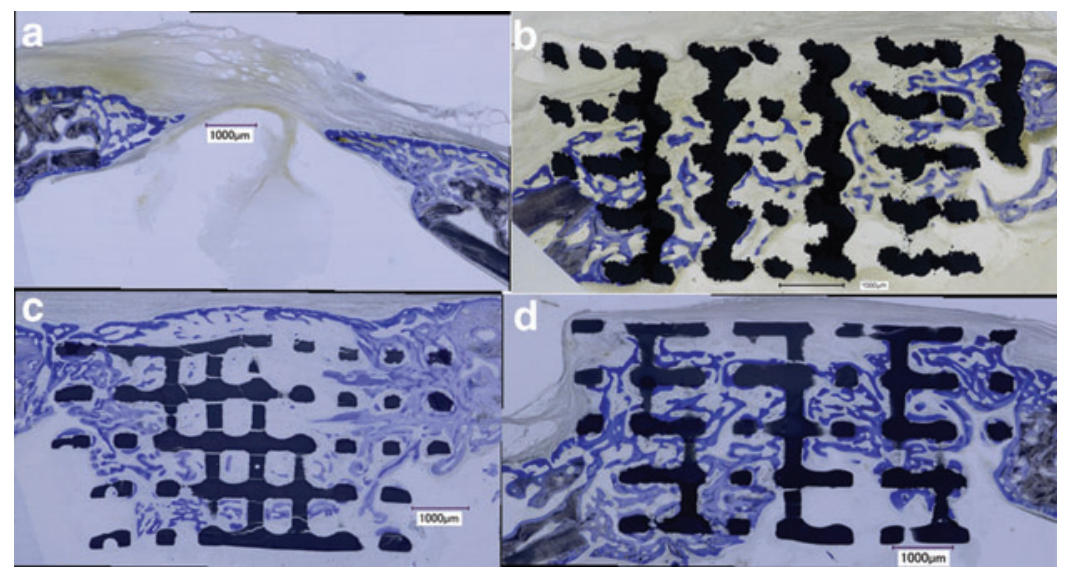

stresses arising from press-fit application of the implants can lead to partial disintegration of the TCP scaffold already during the placement of the implant.

Another important factor for this behavior is that materials such as TCP inherently have different material properties compared to metals. For future studies, this needs to be accounted for by choosing a scaffold design compliant with the mechanical properties of TCP, in particular if applied in critical-size defects. In empty critical $15 \mathrm{~mm}$ defects, $40.13 \% \pm 16.39 \%$ of the middle section was bony bridged after 16 weeks in vivo, $92.13 \% \pm 11.45 \%$ for titanium, and $66.93 \% \pm 14.19 \%$ for TCP, respectively. In the AOI, the percentage of bony regeneration in the middle section was $23.72 \% \pm 10.02 \%$ for empty defects, $42.67 \% \pm 7.38 \%$ for titanium, and $31.51 \% \pm 7.54 \%$ for TCP, respectively. Compared to the untreated defect, bony regenerated area was significantly improved for titanium implants only (Fig. 5d). Bony bridging, however, was significantly improved for both biomaterials, titanium and TCP (Fig. 5e). In direct comparison, the percentage of bony bridging of the defect was significantly higher in titanium scaffolds than in TCP scaffolds. This is seen as clear indication that in the case of a critical-size defect, the mechanical strength of the scaffolds has to be ensured. Since the applied microarchitecture of the titanium scaffold was sufficient to heal the critical-size defect, an additional stimulation by BMP-2 was not tested in this model system.

\section{Discussion}

Cranial defect models are widely used for the testing of bone substitute materials. ${ }^{25-27}$ Less known is the fact that cranial defects are clinically highly relevant in congenital anomalies, trauma, stroke, aneurysms, and cancer. ${ }^{28}$ For preclinical testing, the low mechanical challenge posed in the cranium is an advantage, since it allows the testing of diverse materials without the need for costly fracture fixation devices, needed in long bone defect treatments.

Additive manufacturing facilitates the realization of 3D objects in microarchitectural designs coded in computer files, predominantly in STL format (STereoLithography or Surface TrianguLated). In this study, we manufactured bone substitute scaffolds from TCP by a lithography-based methodology, characterized them, and compared their performance in vivo with titanium-based scaffolds produced with the identical STL file by selective laser melting. The results showed that in a noncritical defect, both scaffolds with identical microarchitecture, but from distinct biomaterials and surfaces performed equally well. In a critical-size
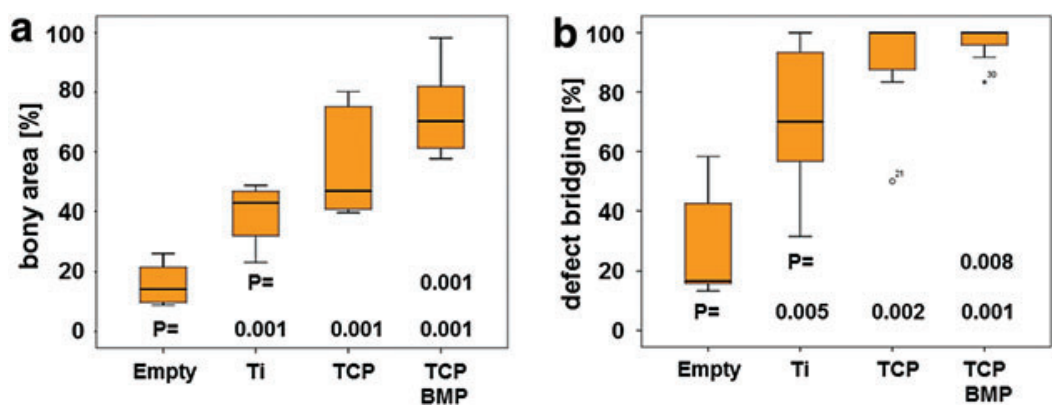

FIG. 4. Histomorphometric parameters for bone regeneration in noncritical-size defects 4 weeks postoperatively. The formation of new bone (a) and defect bridging (b) is significantly elevated in defects treated with scaffolds compared to empty defects. Titanium and TCP scaffolds with identical architecture performed equally well in both categories. A significant increase could only be detected between the titanium and the TCP scaffolds when the latter was doped with $10 \mu \mathrm{g}$ BMP-2. Values are displayed as box plots ranging from the 25th (lower quartile) to the 75th (upper quartile) percentile, including the median as solid black line and whiskers, showing the minimum and maximum values. Values outside the range of the box blot are shown as individual points. $p$ Values are provided in the graphs. Color images available online at www.liebertpub.com/tea 

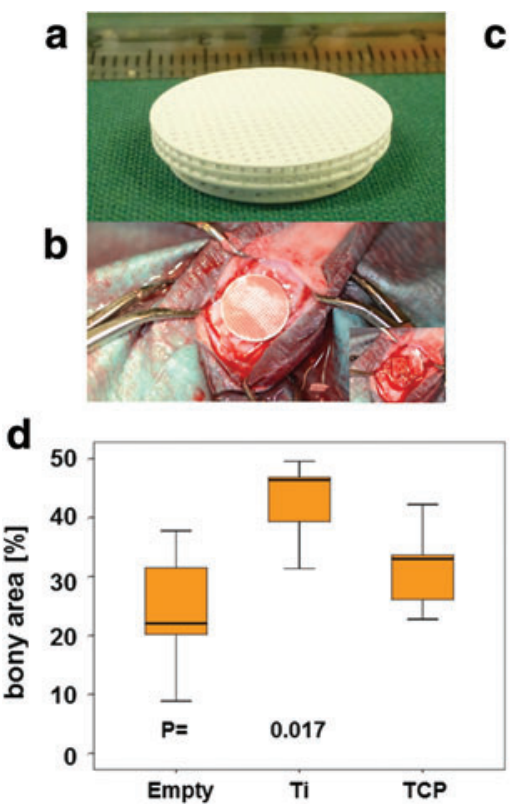

C
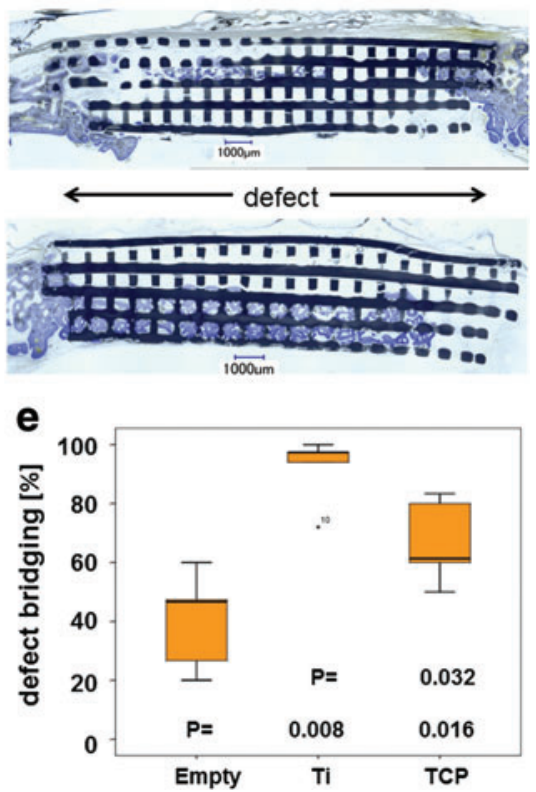

FIG. 5. Critical-size defect. (a) TCP-based implant for critical-size rabbit calvarial bone defect. (b) Intraoperative view on an implanted scaffold. (c) Histological section of the middle of the defect 16 weeks postoperatively. The TCP appears grayish black and bone purple. The distorted lattice structure and gaps between rods indicate a failure of the scaffold. The formation of new bone (d) and defect bridging (e) is significantly elevated in defects treated with titanium scaffolds compared to empty defects. For TCP scaffolds with identical architecture, only defect bridging was significantly improved compared to empty, untreated defects. While no significant difference was evident for bony area between both materials, for bony bridging, titanium performed significantly better than TCP. Values in (d, e) are displayed as box plots ranging from the 25th (lower quartile) to the 75th (upper quartile) percentile, including the median as solid black line and whiskers, showing the minimum and maximum values. Values outside the range of the box blot are shown as individual points. $p$ Values are provided in the graphs. Color images available online at www.liebertpub.com/tea

defect, where the mechanical demand is higher, the titanium-based scaffold was superior, most likely due to the higher mechanical strength of titanium compared to TCP. Thus, for critical-size defects, the brittleness of TCP-based scaffolds will have to be compensated by using different design guidelines for TCP scaffolds as suitable for titanium.

Creeping substitution of the implant over time by bone tissue is the ideal endpoint of a bone regeneration procedure. Therefore, we looked into additive manufacturing of TCP, since it remains biodegradable even after undergoing high temperature sintering. ${ }^{29,30}$ For the production of personalized bone substitutes, the produced implant has to match the digital information deduced from computer tomographies. The lithography-based production of TCP scaffolds by the CeraFab 7500 system matches the lateral dimension of the planned STL file information. However, the height of the pores and rods along the building direction appear compressed (Fig. 1e). The deviation in $z$-direction derives from a curing depth that is higher than the thickness of an individual layer. This increased cure depth is necessary to ensure good adhesion between adjacent layers. The viscosity of the slurry could also play a minor role in this aspect. Deviation in the $z$-axis can be corrected before production by software compensation algorithms to adjust the detailed geometry of the STL file. Moreover, for scaffolds meant for bone tissue engineering, a high precision of the microarchitecture might not be the decisive factor, as long as the structure is widely open, porous, accessible for proteins, blood, and cells, and optimized for bone ingrowth by os- teoconduction. This has been shown when a library of diverse open porous titanium scaffolds was tested in vivo in the same model system. ${ }^{20}$ The outer dimension, however, should perfectly match the defect, to produce personalized bone substitutes, which is the main advantage of the application of additive manufacturing for bone substitute production, especially if complex shapes have to be realized. ${ }^{31}$

Titanium and TCP, both are known for their suitability to serve as bone substitute biomaterial. ${ }^{32}$ In this study, we show that if scaffolds from titanium and TCP are produced with the identical microarchitecture as wide open porous lattice structures, both materials support osteoconduction, ${ }^{20}$ defined in this study as bone ingrowth into porous structures (Fig. 4a) and as guiding cue to achieve defect bridging (Fig. 4b). Therefore, with the lithography-based additive manufacturing procedure, as used in this study, personalized osteoconductive scaffolds can be produced from TCP and other permanent or biodegradable ceramics like hydroxyapatite or bioglass. Since these ceramics vary in their mechanical characteristics, the bone substitute scaffolds can be adjusted to the mechanical need at the operation site with the right choice of the ceramic without changing the microarchitecture or macroarchitecture.

Recent studies have reported on bone ingrowth and the presence of cells also in micropores, well below $0.1 \mathrm{~mm}$ in diameter. ${ }^{33,34}$ The positive effect of microporosity on bone formation was suggested to reflect a better attachment of proteins to the surface, increased degradation products, and capillary forces (as reviewed in Ref. ${ }^{35}$ ). In our study, we 
compared a microporous TCP-based scaffold (Fig. 2) to a material without pores (titanium) and could not detect a significant difference in defect bridging or regenerated bony area for wide open porous lattice structures. This is in line with other in vivo studies in sheep, where different levels of microporosity in TCP-based scaffolds had no effect on bony healing. ${ }^{36}$

To our knowledge, this is the first direct comparison of a metal and a ceramic with the identical microarchitecture in vivo, pointing to the importance of the microarchitecture for osteoconduction and bone regeneration. When using scaffold designs of the exact same geometry, significant differences in terms of the mechanical integrity became visible for the critical-size defects (Fig. 5). These shortcomings of the brittle TCP scaffolds will have to be compensated by tailoring and improving the scaffold designs. It could be verified that a geometry working well for dense and ductile materials such as titanium is not necessarily the best fit for other types of materials, especially outside the material class of metals. For small noncritical defects, TCP-based scaffolds performed excellently. The anticipated difference in degradation capability will have long-time effects and was not subject of this study.

By selecting the right ceramic in combination with the right scaffold microarchitecture, mechanical demands or degradation characteristics can be tuned to the need of individual patients in cranio-maxillofacial surgery, orthopedics, trauma, or dentistry. Especially in terms of developing optimized scaffold designs, which are compliant with the property profile of ceramics such as TCP, additional research is necessary to improve the performance of such implants.

\section{Acknowledgments}

We thank Ana Perez, Indranil Bhattacharya, and AlexandreTchouboukov for excellent technical assistance. All animal work was kindly supported by Flora Nichols and Thea Fleischmann. We thank Martin Schwentenwein and Johannes Homa for help during the design and the initial production phase of TCP scaffolds. This research work was supported by grants from the Swiss National Science Foundation to F.E.W.

\section{Disclosure Statement}

No competing financial interests exist.

\section{References}

1. Growing New Bone for More Effective Injury Repair. Medicine and Health/Medical Research, Isle Of Man, United Kingdom: Medical Press, 2017.

2. Hollister, S.J., and Murphy, W.L. Scaffold translation: barriers between concept and clinic. Tissue Eng B Rev 17, 459, 2011.

3. Bianco, P., Robey, P.G., and Simmons, P.J. Mesenchymal stem cells: revisiting history, concepts, and assays. Cell Stem Cell 2, 313, 2008.

4. Brennan, M.Á, Renaud, A., Amiaud, J., et al. Pre-clinical studies of bone regeneration with human bone marrow stromal cells and biphasic calcium phosphate. Stem Cell Res Ther 5, 114, 2014.
5. Cancedda, R., Dozin, B., Giannoni, P., and Quarto, R. Tissue engineering and cell therapy of cartilage and bone. Matrix Biol 22, 81, 2003.

6. Mehrkens, A., Saxer, F., Güven, S., et al. Intraoperative engineering of osteogenic grafts combining freshly harvested, human adipose-derived cells and physiological doses of bone morphogenetic protein-2. Eur Cells Mater 24, 308, 2012.

7. Carragee, E.J., Hurwitz, E.L., and Weiner, B.K. A critical review of recombinant human bone morphogenetic protein2 trials in spinal surgery: emerging safety concerns and lessons learned. Spine J 11, 471, 2011.

8. Martin, B.I., Lurie, J.D., Tosteson, A.N.A., Deyo, R.A., Farrokhi, F.R., and Mirza, S.K. Use of bone morphogenetic protein among patients undergoing fusion for degenerative diagnoses in the United States, 2002-2012. Spine J 15, 692, 2015.

9. Urist, M.R. Bone: formation by autoinduction. Science 150, 893,1965

10. James, A.W., LaChaud, G., Shen, J., et al. A review of the clinical side effects of bone morphogenetic protein-2. Tissue Eng B Rev 22, 284, 2016.

11. Galván-Chacón, V.P., and Habibovic, P. Deconvoluting the bioactivity of calcium-phosphate-based bone graft substitutes: Strategies to understand the role of individual material properties. Adv Healthcare Mater 6, 1601478, 2017.

12. Chai, Y.C., Carlier, A., Bolander, J., et al. Current views on calcium phosphate osteogenicity and the translation into effective bone regeneration strategies. Acta Biomater 8, 3876, 2012.

13. Karageorgiou, V., and Kaplan, D. Porosity of 3D biomaterial scaffolds and osteogenesis. Biomaterials 26, 5474, 2005.

14. von Doernberg, M.C., von Rechenberg, B., Bohner, M., et al. In vivo behavior of calcium phosphate scaffolds with four different pore sizes. Biomaterials 27, 5186, 2006.

15. Brunello, G., Sivolella, S., Meneghello, R., et al. Powderbased 3D printing for bone tissue engineering. Biotechnol Adv 34, 740, 2016.

16. Sachs, E., Cima, M., and Cornie, J. Three-dimensional printing: rapid tooling and prototypes directly from a CAD Model. CIRP Ann 39, 201, 1990.

17. Butscher, A., Bohner, M., Roth, C., et al. Printability of calcium phosphate powders for three-dimensional printing of tissue engineering scaffolds. Acta Biomater 8, 2012.

18. Butscher, A., Bohner, M., Doebelin, N., Hofmann, S., and Müller, R. New depowdering-friendly designs for threedimensional printing of calcium phosphate bone substitutes. Acta Biomater 9, 9149, 2013.

19. de Wild, M., Schumacher, R., Mayer, K., et al. Bone regeneration by the osteoconductivity of porous titanium implants manufactured by selective laser melting: a histological and $\mu \mathrm{CT}$ study in the rabbit. Tissue Eng A 19, 2645, 2013.

20. de Wild, M., Zimmermann, S., Rüegg, J., et al. Influence of microarchitecture on osteoconduction and mechanics of porous titanium scaffolds generated by selective laser melting. 3D Print Addit Manuf 3, 142, 2016.

21. Karfeld-Sulzer, L.S., Ghayor, C., Siegenthaler, B., de Wild, M., Leroux, J.C., and Weber, F.E. N-methyl pyrrolidone/ bone morphogenetic protein-2 double delivery with in situ forming implants. J Control Release 203, 181, 2015.

22. Schwentenwein, M., and Homa, J. Additive manufacturing of dense alumina ceramics. Int J Appl Ceram Technol 12, 1, 2015. 
23. Kruse, A., Jung, R.E., Nicholls, F., Zwahlen, R.A., Hämmerle, C.H., and Weber, F.E. Bone regeneration in the presence of a synthetic hydroxyapatite-silica oxide-based and a xenogenic hydroxyapatite-based bone substitute material. Clin Oral Implants Res 22, 506, 2011.

24. Schmidlin, P.R., Nicholls, F., Kruse, A., Zwahlen, R.A., and Weber, F.E. Evaluation of moldable, in situ hardening calcium phosphate bone graft substitutes. Clin Oral Implants Res 24, 149, 2013.

25. Hollinger, J.O., and Kleinschmidt, J.C. The critical size defect as an experimental model to test bone repair materials. J Craniofac Surg 1, 60, 1990.

26. Kim, J., Magno, M.H., Waters, H., et al. Bone regeneration in a rabbit critical-sized calvarial model using tyrosine-derived polycarbonate scaffolds. Tissue Eng A 18, 1132, 2012.

27. Spicer, P.P., Kretlow, J.D., Young, S., Jansen, J.A., Kasper, F.K., and Mikos, A.G. Evaluation of bone regeneration using the rat critical size calvarial defect. Nat Protoc 7, 1918, 2012.

28. Lee, J.C., and Volpicelli, E.J. Bioinspired collagen scaffolds in cranial bone regeneration: from bedside to bench. Adv Healthc Mater 6, $1700232,2017$.

29. Wiltfang, J., Merten, H.A., Schlegel, K.A., et al. Degradation characteristics of $\alpha$ and $\beta$ tri-calcium-phosphate (TCP) in minipigs. J Biomed Mater Res 63, 115, 2002.

30. Yamada, M., Shiota, M., Yamashita, Y., and Kasugai, S. Histological and histomorphometrical comparative study of the degradation and osteoconductive characteristics of $\alpha$ and $\beta$-tricalcium phosphate in block grafts. J Biomed Mater Res B 82, 139, 2007.

31. Seitz, H., Rieder, W., Irsen, S., Leukers, B., and Tille, C. Three-dimensional printing of porous ceramic scaffolds for bone tissue engineering. J Biomed Mater Res B 74, $782,2005$.
32. Tevlin, R., McArdle, A., Atashroo, D., et al. Biomaterials for craniofacial bone engineering. J Dent Res 93, 1187, 2014.

33. Bernstein, A., Niemeyer, P., Salzmann, G., et al. Microporous calcium phosphate ceramics as tissue engineering scaffolds for the repair of osteochondral defects: histological results. Acta Biomater 9, 7490, 2013.

34. Polak, S.J., Rustom, L.E., Genin, G.M., Talcott, M., and Wagoner Johnson, A.J. A mechanism for effective cellseeding in rigid, microporous substrates. Acta Biomater 9, 7977, 2013.

35. Zhang, K., Fan, Y., Dunne, N., and Li, X. Effect of microporosity on scaffolds for bone tissue engineering.Regen Biomater rby 001, 115, 2018.

36. Lapczyna, H., Galea, L., Wüst, S., et al. andvon Rechenberg, B. Effect of grain size and microporosity on the in vivo behaviour of beta-tricalcium phosphate scaffolds. Eur Cells Mater 28, 299, 2014.

Address correspondence to: Franz E. Weber, PhD Department of Cranio-Maxillofacial and Oral Surgery Oral Biotechnology and Bioengineering Center for Dental Medicine University of Zürich Plattenstrasse 11 8032 Zürich Switzerland

E-mail: franz.weber@zzm.uzh.ch

Received: January 17, 2018 Accepted: April 27, 2018

Online Publication Date: August 27, 2018 